\title{
The relationship between steps of 6MWT and COPD severity: a cross-sectional study
}

This article was published in the following Dove Medical Press journal: International Journal of COPD

\author{
Guan-Sheng Zeng* \\ Li-Chang Chen* \\ Hui-Zhen Fan \\ Ling-Ling $\mathrm{Wu}$ \\ Xi-Ping Wu \\ Ze-Kui Fang \\ $\mathrm{Xi} \mathrm{He}$ \\ Hua-Peng Yu
}

Department of Pulmonary and Critical Care Medicine, Zhujiang Hospital, Southern Medical University,

Guangzhou, China

*These authors contributed equally to this work
Correspondence: Hua-Peng Yu Department of Pulmonary and Critical Care Medicine, Zhujiang Hospital of Southern Medical University, No. 253, Industrial Avenue, Guangzhou, Guangdong 510280, China

$\mathrm{Tel}+8602061643175$

Fax +8602084306143

Email huapengyu@aliyun.com
Background and objective: The distance of 6-minute walk test (D6MWT) has been widely used in the assessment of functional status in patients with COPD, while very little attention has been paid to the role of steps of 6-minute walk test (S6MWT). The purpose of this study was to investigate the relationship between S6MWT and other physiologic parameters of COPD.

Patients and methods: Seventy patients with stable COPD were enrolled consecutively in this cross-sectional study. Pulmonary function tests, including spirometry, impulse oscillometry (IOS) and the single-breath diffusing capacity of the lungs for carbon monoxide $\left(\mathrm{DL}_{\mathrm{CO}}\right)$, were carried out at rest. Quality of life was assessed by health-related quality of life (HRQoL) questionnaires, including modified Medical Research Council dyspnea scale (mMRC), St George's Respiratory Questionnaire, Chronic Obstructive Pulmonary Disease Assessment Test (CAT) and Clinical Chronic Obstructive Pulmonary Questionnaire. Both steps and distance were measured in the following 6-minute walk test (6MWT).

Results: Both S6MWT and D6MWT showed significant correlation with spirometry, IOS, $\mathrm{DL}_{\mathrm{CO}}$ parameters and HRQoL questionnaires score. Both pre- and post-6MWT inspiratory capacity showed significant correlation with S6MWT $(\rho=0.338, P=0.004 ; \rho=0.359$, $P=0.002$, respectively), whereas did not correlate with D6MWT $(\rho=0.145, P=0.230 ; \rho=0.160$, $P=0.189$, respectively). In stepwise multiple regression analysis, mMRC grade, age and CAT score remained as significant predictors in the final model for D6MWT (adjusted $R^{2}=0.445$, $P<0.01)$. $\mathrm{DL}_{\mathrm{CO}}$ and CAT score remained as significant predictors in the final model for S6MWT (adjusted $R^{2}=0.417, P<0.01$ ).

Conclusion: S6MWT is efficient in the evaluation of functional status and quality of life in COPD and has significant correlation with various parameters indicating disease severity. Additionally, S6MWT might be better in predicting lung hyperinflation in COPD compared with D6MWT.

Keywords: COPD, steps, 6MWT, hyperinflation

\section{Introduction}

COPD is characterized by persistent airflow limitation and decline in exercise tolerance. ${ }^{1,2}$ Patient management of COPD demands regular assessment of the patients' functional status. Compared with other alternative exercise tests including cardiopulmonary exercise test and the shuttle walk test, the 6-minute walk test (6MWT) is a relatively simple and economical method for assessing the functional status of cardiopulmonary disease and has been widely used in patient management of COPD. ${ }^{3}$ In a number of studies, distance of the 6-minute walk test (D6MWT) has been regarded as an important index of exercise tolerance in patients with COPD and correlates with mortality. ${ }^{4-7}$ Nevertheless, so far, very little attention has been paid to the role of steps of 6-minute walk test (S6MWT). Whether S6MWT is associated with 
severity of COPD remains unclear. It has been demonstrated that gait patterns or concern of steps in daily life influence pulmonary function parameters and quality of life in patients with COPD; ${ }^{8,9}$ therefore, we hypothesized that the total steps of 6MWT may also be an efficient indicator of exercise tolerance and well correlated with traditional indexes of COPD.

The main purpose of the present study was to investigate the relationship of total steps during 6MWT with traditional indicators of COPD severity, including pulmonary function parameters, quality of life and also undertake comparison with D6MWT. Besides, since data from prior study suggested that dynamic lung hyperinflation occurs in 6MWT, ${ }^{10}$ leading to changes in spirometry such as inspiratory capacity (IC), both pre- and post-6MWT spirometry parameters were measured and involved in the statistical analysis as a contrast.

\section{Patients and methods}

This was a cross-sectional study conducted in Zhujiang Hospital of Southern Medical University, Guangzhou, China. A total of 70 patients with stable COPD were enrolled consecutively in the study between June 2018 and September 2018. The clinical diagnosis of COPD was a ratio of postbronchodilator $\mathrm{FEV}_{1} / \mathrm{FVC}<70 \%$ according to the GOLD 2018 guidelines. ${ }^{11}$ Participants with an exacerbation within 4 weeks prior to the tests or any severe comorbidity including unstable cardiac or pulmonary disease were excluded. Participants were required to continue with their regular therapy before performing the tests of the study. Pulmonary function tests, including spirometry, impulse oscillometry (IOS) and the single-breath diffusing capacity of the lungs for carbon monoxide $\left(\mathrm{DL}_{\mathrm{CO}}\right)$, were carried out before the 6MWT. Quality of life assessed by questionnaires, oxygen saturation and pulse at resting state were also measured. Then, we conducted the 6MWT and recorded both the distance and total steps during the 6 minutes. Immediately after the $6 \mathrm{MWT}$, the spirometry, pulse and oxygen saturation were measured again for additional post-6MWT parameters.

\section{Pulmonary function tests}

Pulmonary function tests were performed according to the guidances of European Respiratory Society/American Thoracic Society (ATS) using Jaeger Masterscreen Device (E. Jaeger GmbH, Wurzburg, Germany). ${ }^{12}$ Pulmonary hyperinflation was reflected by $\mathrm{IC}$ and $\mathrm{DL}_{\mathrm{CO}}$. IC was performed according to the guidelines described by Guenette et al. ${ }^{13}$ After four to six tidal breath, participants at functional residual capacity were encouraged to breathe in as much air as possible to total lung capacity (TLC), and then exhaled all the air to residual volume $(\mathrm{RV}) . \mathrm{DL}_{\mathrm{CO}}$ was measured by single-breath washout method. The participants were asked to blow out all of the air that they can, leaving only the residual lung volume of gas. Then, he inhaled a test gas mixture rapidly and completely, reaching the TLC as nearly as possible. After holding in the lung for about 10 seconds, the gas was exhaled. ${ }^{14}$ IOS and $\mathrm{DL}_{\mathrm{CO}}$ were performed only once before the 6MWT. Spirometry was performed before the 6MWT and immediately after the walking test.

\section{Quality of life}

Health-related quality of life (HRQoL) questionnaires were applied in the assessment of quality of life. ${ }^{11}$ We used four different questionnaires in all, including modified Medical Research Council dyspnea scale (mMRC), St George's Respiratory Questionnaire (SGRQ), Chronic Obstructive Pulmonary Disease Assessment Test (CAT) and Clinical Chronic Obstructive Pulmonary Questionnaire (CCQ). The mMRC utilizes a scale with a grade ranging from 0 to 4 to evaluate the degree of dyspnea in patients with COPD. ${ }^{15}$ SGRQ score ranges from 0 to 100 , it is the most widely used questionnaire developed to measure impaired health and quality of life in airways disease. ${ }^{16}$ The CAT, consisting of eight items, is a short questionnaire designed to measure symptoms and impacts of COPD. ${ }^{17} \mathrm{CCQ}$ is another short questionnaire used to quantify symptom burden and can be divided into three parts of symptoms, functional and mental effects. ${ }^{18}$ Participants were asked to choose the description most consistent with their dyspnea symptoms or rate how strongly they agreed with each statement. For all the four questionnaires, higher grade or score represented lower quality of life and worse health status.

\section{The 6MWT}

The 6MWT was carried out in a flat, 30-m-long corridor. A traffic cone was placed at the turnaround point as a mark. Participants were asked to walk back and forth as far as possible on the corridor in a period of 6 minutes. They were allowed to rest if extremely fatigue and encouraged to continue the walking as soon as possible. Encouraging phrases recommended by the official statement of ATS were given by a researcher who counted the lap number at the same time. ${ }^{19}$ The steps during the test were counted manually and individually by two other researchers.

\section{Statistical analyses}

All the data management and statistical analyses were performed using IBM SPSS Statistics 19.0 (IBM Corporation, 
Armonk, NY, USA). Descriptive data were generated for all variables. Results were expressed in the form of mean $\pm \mathrm{SD}$ or median (IQR 25-75) as appropriate. Difference of pulmonary function between pre- and post-6MWT was tested by paired $t$-test or Wilcoxon signed-rank test based on the distribution of data. Relationship of pulmonary function parameters and quality of life with distance or steps, respectively, of the 6MWT was assessed by Spearman's rank correlation coefficient. The correlation coefficient was expressed as $\rho$. Covariates showed that significant relationship was retained as potential factors in the following multiple regression analysis. The multiple linear regression analysis was carried out to examine the deeper relationship between distance or steps and numerous variates using stepwise regression algorithm and only pre-6MWT parameters were adopted in this analysis. For the distance of 6MWT, age, $\mathrm{FVC}, \mathrm{FEV}_{1}, \mathrm{DL}_{\mathrm{CO}}$, ratio of RV to TLC, reactance at $20 \mathrm{~Hz}\left(R_{20}\right)$, reactance at $5 \mathrm{~Hz}$ $\left(\mathrm{X}_{5}\right)$, mMRC grade, SGRQ score, CAT score and CCQ score were included as covariates in the model. As for the steps of 6MWT, IC, FVC, $\mathrm{FEV}_{1}, \mathrm{SpO}_{2}, \mathrm{DL}_{\mathrm{CO}}$, RV/TLC, $\mathrm{X}_{5}, \mathrm{mMRC}$ grade, SGRQ score, CAT score and CCQ score were included as covariates. A two-sided $P$-value $<0.05$ was considered to be statistically significant. Bonferroni correction was applied to determine a new alpha level in the correlational analysis.

\section{Ethics statement}

This study was conducted in accordance with the Declaration of Helsinki. The procedures of this study were approved by the ethics committee of Zhujiang Hospital of Southern Medical University under code 2018-HXNK-001. Participants were informed of the potential risks of the test, and written informed consents were obtained from all the participants.

\section{Results}

Characteristics of the subjects are presented in Table 1. Seventy participants were recruited and $80 \%$ of them were male. The median age of all the participants was 63.0 years (IQR 25-75, 54.0-68.0 years). Based on the GOLD 2018 severity classification, $22(31.4 \%)$ of them were mild, $32(45.7 \%)$ moderate, $11(11.5 \%)$ severe and $5(7.1 \%)$ very severe. ${ }^{11}$ When based on the GOLD ABCD Group classification, $3(4.3 \%)$ of them were in Group A, $47(67.1 \%)$ in Group B, 3 (4.7\%) in Group C and 17 (24.3\%) in Group D. Sixty-one (87.1\%) participants were under regular treatment with bronchodilator. The median of D6MWT was $533.0 \mathrm{~m}$ (IQR 25-75, 495.8-562.5 m) and the median of S6MWT was 739 steps (IQR 25-75, 701-756 steps). Table 2 demonstrates the differences between pre- and post-6MWT spirometry
Table I Demographic characteristics, pulmonary function and HRQoL questionnaire scores of 70 COPD patients

\begin{tabular}{l|l}
\hline \multirow{2}{*}{ Characteristics } & Total \\
\cline { 2 - 2 } & $(\mathbf{n}=\mathbf{7 0})$ \\
\hline Age (years) & $63.0(54.0-68.0)$ \\
$\mathrm{BMI}\left(\mathrm{kg} / \mathrm{m}^{2}\right)$ & $22.8(20.9-24.8)$ \\
$\mathrm{DL}_{\mathrm{co}}(\%$ predicted $)$ & $76.7(55.6-91.5)$ \\
$\mathrm{TLC}(\%$ predicted) & $86.3 \pm \mathrm{II} .1$ \\
$\mathrm{RV}(\%$ predicted $)$ & $92.2(80.0-109.3)$ \\
$\mathrm{RV} / \mathrm{TLC}(\%)$ & $42.1 \pm 9.3$ \\
$R_{5}(\mathrm{kPa} / \mathrm{L} / \mathrm{s})$ & $0.4(0.3-0.5)$ \\
$R_{20}(\mathrm{kPa} / \mathrm{L} / \mathrm{s})$ & $0.3 \pm 0.1$ \\
$\mathrm{X}_{5}(\mathrm{kPa} / \mathrm{L} / \mathrm{s})$ & $-0.1(-0.2$ to -0.1$)$ \\
$\mathrm{mMRC}$ grade & $0.0(0.0-1.0)$ \\
SGRQ score & $17.5(9.0-36.0)$ \\
CCQ score & $1.6(1.1-2.3)$ \\
CAT score & $13.5(\mathrm{I} .0-18.3)$ \\
D6MWT (m) & $533.0(495.8-562.5)$ \\
S6MWT (step) & $739(70 \mathrm{I}-756)$ \\
\hline
\end{tabular}

Note: Results were expressed in the form of mean \pm SD or median (IQR 25-75) as appropriate.

Abbreviations: BMI, body mass index; CAT, Chronic Obstructive Pulmonary Disease Assessment Test; CCQ, Clinical Chronic Obstructive Pulmonary Questionnaire; $\mathrm{D} 6 \mathrm{MWT}$, distance of 6-minute walk test; $\mathrm{DL}_{\mathrm{co}}$, diffusing capacity of the lungs for carbon monoxide; HRQoL, health-related quality of life; mMRC, modified Medical Research Council dyspnea scale; $R_{20}$, reactance at $20 \mathrm{~Hz} ; R_{5}$, reactance at $5 \mathrm{~Hz}$; RV, residual volume; S6MWT, steps of 6MWT; SGRQ, St George's Respiratory Questionnaire; TLC, total lung capacity; $X_{5}$, reactance at $5 \mathrm{~Hz}$.

parameters. IC, $\mathrm{FEV}_{1} / \mathrm{FVC}, \mathrm{SpO}_{2}$ and pulse increased significantly after the 6MWT.

\section{Relationship between distance or steps and other parameters}

The results of the correlational analysis between D6MWT or S6MWT, pulmonary function parameters (spirometry, IOS and $\mathrm{DL}_{\mathrm{CO}}$ ) and quality of life (mMRC, SGRQ, CAT and CCQ) are summarized in Table 3. Among general characteristics, age showed a significantly negative correlation with D6MWT ( $\rho=-0.485, P<0.001)$, but not with S6MWT $(\rho=-0.229$, $P=0.057)$. Gender proportion and body mass index did not show significant correlation with neither distance nor steps.

Table 2 Comparison between pre- and post-6MWT pulmonary function

\begin{tabular}{l|l|l|l}
\hline & Pre-6MWT & Post-6MWT & P-value \\
\hline IC (\% predicted) & $79.6 \pm 19.1$ & $81.1(69.3-97.0)$ & $0.03 I^{*}$ \\
FVC (\% predicted) & $88.3 \pm 19.3$ & $88.0 \pm 18.4$ & 0.694 \\
FEV $_{1}(\%$ predicted) & $68.2 \pm 23.5$ & $68.3 \pm 23.4$ & 0.917 \\
$\mathrm{FEV}_{1} / \mathrm{FVC}(\%)$ & $56.8(42.4-65.2)$ & $57.6(46.0-66.3)$ & $<0.00 \mathrm{I}^{*}$ \\
$\mathrm{SPO}_{2}(\%)$ & $96.0(95.0-97.0)$ & $97.0(96.0-98.0)$ & $0.020^{*}$ \\
Pulse (n/min) & $78.0(70.8-88.3)$ & $98.9 \pm 15.2$ & $<0.00 I^{*}$ \\
\hline
\end{tabular}

Notes: *Statistical significance. Results were expressed in the form of mean $\pm S D$ or median (IQR 25-75) as appropriate.

Abbreviations: 6MWT, 6-minute walk test; IC, inspiratory capacity. 
Table 3 Relationship between D6MWT or S6MWT and other parameters

\begin{tabular}{|c|c|c|c|c|}
\hline \multirow[t]{2}{*}{ Functional parameters } & \multicolumn{2}{|c|}{ D6MWT (m) } & \multicolumn{2}{|c|}{ S6MWT (step) } \\
\hline & $\rho$ & $P$-value & $\rho$ & $P$-value \\
\hline Age (years) & -0.485 & $<0.00 I^{*}$ & -0.229 & 0.057 \\
\hline Pre-6MWT IC (\% predicted) & 0.145 & 0.230 & 0.338 & $0.004 *$ \\
\hline Post-6MWT IC (\% predicted) & 0.160 & 0.189 & 0.359 & $0.002 *$ \\
\hline Pre-6MWT FVC (\% predicted) & 0.305 & $0.010^{*}$ & 0.363 & $0.002^{*}$ \\
\hline Post-6MWT FVC (\% predicted) & 0.267 & $0.025^{*}$ & 0.296 & $0.013^{*}$ \\
\hline Pre-6MWT FEV $(\%$ predicted) & 0.330 & $0.005^{*}$ & 0.426 & $<0.00 I^{*}$ \\
\hline Post-6MWT FEV (\% predicted) & 0.310 & $0.009 *$ & 0.388 & $0.001 *$ \\
\hline Pre-6MWT FEV,/FVC (\%) & 0.373 & $0.001 *$ & 0.406 & $<0.00 I^{*}$ \\
\hline Post-6MWT FEV,/FVC (\%) & 0.293 & $0.014^{*}$ & 0.373 & $0.001 *$ \\
\hline Pre-6MWT SpO 2 (\%) & 0.182 & 0.132 & 0.289 & $0.015^{*}$ \\
\hline Post-6MWT $\mathrm{SpO}_{2}(\%)$ & 0.245 & $0.04 I^{*}$ & 0.201 & 0.095 \\
\hline $\mathrm{DL}_{\mathrm{co}}(\%$ predicted $)$ & 0.411 & $<0.001 *$ & 0.548 & $<0.00 I^{*}$ \\
\hline $\mathrm{RV} / \mathrm{TLC}(\%)$ & -0.326 & $0.006 *$ & -0.286 & $0.016 *$ \\
\hline$R_{20}(\mathrm{kPa} / \mathrm{L} / \mathrm{s})$ & 0.244 & $0.042^{*}$ & 0.171 & 0.157 \\
\hline $\mathrm{X}_{5}(\mathrm{kPa} / \mathrm{L} / \mathrm{s})$ & 0.264 & $0.027^{*}$ & 0.336 & $0.004^{*}$ \\
\hline mMRC grade & -0.439 & $<0.001 *$ & $-0.45 I$ & $<0.00 I^{*}$ \\
\hline SGRQ score & -0.332 & $0.005^{*}$ & -0.344 & $0.004^{*}$ \\
\hline CCQ score & -0.435 & $<0.001 *$ & -0.370 & $0.002 *$ \\
\hline CAT score & -0.393 & $0.00 I^{*}$ & -0.431 & $<0.00 I^{*}$ \\
\hline
\end{tabular}

Note: *Statistical significance.

Abbreviations: CAT, Chronic Obstructive Pulmonary Disease Assessment Test; CCQ, Clinical Chronic Obstructive Pulmonary Questionnaire; D6MWT, distance of 6-minute walk test; $\mathrm{DL}_{\mathrm{co}}$, diffusing capacity of the lungs for carbon monoxide; IC, inspiratory capacity; mMRC, modified Medical Research Council dyspnea scale; $\mathrm{RV}$, residual volume; $R_{20}$, reactance at $20 \mathrm{~Hz}$; S6MWT, steps of 6MWT; SGRQ, St George's Respiratory Questionnaire; TLC, total lung capacity; $\rho$, correlation coefficient; $\mathrm{X}_{5}$, reactance at $5 \mathrm{~Hz}$.

The relationship of D6MWT or S6MWT with spirometry parameters was similar, both before and after the 6MWT. All of pre- and post-6MWT FVC, pre- and post-6MWT FEV , $_{1}$ pre- and post-6MWT FEV $\mathrm{F}_{1} / \mathrm{FVC}$ were significantly positively correlated with distance and steps. As regards IC, both preand post-6MWT IC showed significant correlation with steps ( $\rho=0.338, P=0.004 ; \rho=0.359, P=0.002$, respectively), whereas the same did not correlate with distance $(\rho=0.145$, $P=0.230 ; \rho=0.160, P=0.189$, respectively) (Figure 1). Neither pre-6MWT nor post-6MWT pulse was correlated with distance or steps.

For $\mathrm{DL}_{\mathrm{CO}}$, IOS parameters and quality of life assessed by questionnaires, a significant positive correlation was observed between $\mathrm{DL}_{\mathrm{CO}}$ and both distance and steps. RV/TLC showed significant correlation with distance and steps, while RV or TLC did not. Among the IOS parameters, $R_{20}$ was only significantly correlated with distance, and $\mathrm{X}_{5}$ was correlated with both distance and steps. A significant negative correlation was found between the score of all the four questionnaires (mMRC, SGRQ, CAT and CCQ) and both D6MWT and S6MWT.

In order to decrease the risk of type I error when making multiple individual correlational analysis, a stricter alpha level of $0.0026(0.05 / 19)$ determined by Bonferroni correction is applied to reappraise the relationship between D6MWT or S6MWT and other parameters additionally. Under the new test level, D6MWT was significantly correlated with age, pre-6MWT FEV ${ }_{1} / \mathrm{FVC}, \mathrm{DL}_{\mathrm{CO}}$, $\mathrm{mMRC}, \mathrm{CCQ}$ and CAT. S6MWT was significantly correlated with pre- and post-6MWT FEV ${ }_{1}$, pre-6MWT FVC, pre- and post-6MWT FEV $_{1} /$ FVC, post-6MWT IC, DL ${ }_{C O}$, mMRC, CCQ and CAT.

\section{Stepwise multiple regression analysis}

Stepwise multiple linear regression analysis was performed to find out the predictors of D6MWT or S6MWT. Potential factors were retained in the multiple linear regression analysis as mentioned above. In the final model for D6MWT, mMRC grade, age and CAT score remained as significant predictors with an adjusted $R^{2}$ of 0.445 (Table 4). With respect to S6MWT, only $\mathrm{DL}_{\mathrm{CO}}$ and CAT score remained as significant predictors in the final model with an adjusted $R^{2}$ of 0.417 (Table 5).

\section{Discussion}

In the current study, we explored the relationship between S6MWT and various indexes indicating the severity of COPD, including spirometry and IOS parameters, $\mathrm{DL}_{\mathrm{CO}}$, oxygen saturation, pulse, mMRC grade, SGRQ score, CAT score and CCQ score. One of the most significant findings to emerge from this study is that S6MWT had a good correlation with pulmonary function parameters and quality of life. Therefore, S6MWT could be used as a novel index in evaluating functional status of patients with COPD, which may be better than D6MWT in some case. Additionally, S6MWT probably suggests lung hyperinflation in COPD.

There were strengths of our study. First, previous studies mainly focused on the status and role of D6MWT, which well represents exercise tolerance, in the evaluation of functional status in COPD. However, S6MWT has never been studied while it may have an interest. As for our research, it had revealed the role of the S6MWT in the assessment of COPD and made comparisons with D6MWT at the same time. Second, for either of the two aspects of COPD, pulmonary function and quality of life, we had used several different methods for measurements (spirometry, IOS, single-breath washout and four respiratory questionnaires) in order to obtain more representative and comprehensive data.

It is widely acknowledged that D6MWT correlates well with pulmonary function parameters. ${ }^{4}$ The correlational analysis results of this study corroborated the findings of the previous work in COPD, which showed significant 

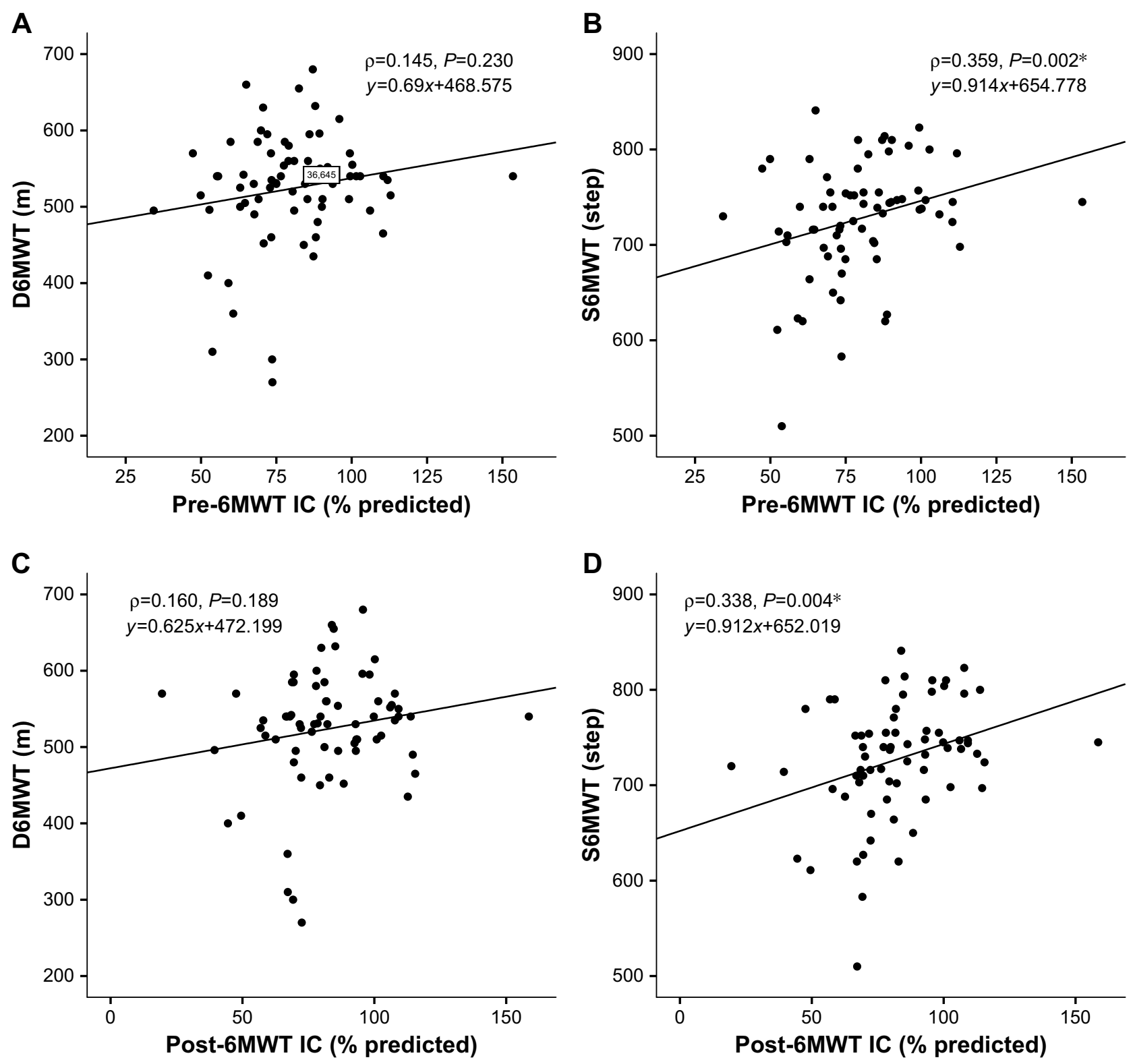

Figure I Correlation between D6MWT or S6MWT and IC in COPD.

Notes: *Statistical significance. Neither pre-6MWT IC (A) nor post-6MWT IC (C) is significantly correlated with distance of 6MWT. Both pre-6MWT IC (B) and post6MWT IC (D) are significantly correlated with steps of 6MWT.

Abbreviations: 6MWT, 6-minute walk test; D6MWT, distance of 6MWT; IC, inspiratory capacity; S6MWT, steps of 6MWT.

correlation between $\mathrm{FEV}_{1}$, FVC or FEV / FVC and D6MWT. The association between S6MWT and spirometry parameters was similar to that of D6MWT, other than IC. Previous research had established that changes in IC reflect the ability of a patient with COPD to increase ventilation in response to the increasing metabolic demands of exercise ${ }^{20}$ but may also indicate the degree of lung hyperinflation. ${ }^{21}$ Our results showed that only steps correlated with IC and not distance walked. This can be explained by considering that steps is affected by lung hyperinflation. However, the precise mechanism remains to be elucidated.
A study by Marin et al showed that pulmonary function changed during daily exercise such as 6MWT and the resting spirometry parameters can only explained part of the dyspnea experienced in daily life. ${ }^{22}$ Further support for this view comes from a small study by Wibmer et al (2014) that showed post-6MWT spirometry measures were better predictors of exercise tolerance than pre-6MWT measures. ${ }^{23}$ Therefore, both pre- and post-6MWT parameters were collected in this study. The results of pre- and post-6MWT spirometry are presented in Tables 2 and 3. Our study reported a similar moderate correlation between pre- and post-6MWT lung 
Table 4 Stepwise multiple linear regression analysis for D6MWT

\begin{tabular}{l|l|l|l|l}
\hline Variables & Coefficient $(95 \%$ CI) & Standardized $\beta$ & $P$-value & VIF \\
\hline Intercept & $741.063(651.726-830.400)$ & & & \\
mMRC grade & $-17.986(-34.754$ to -1.218$)$ & -0.288 & $0.036^{*}$ & 2.245 \\
Age (years) & $-2.328(-3.516$ to -1.140$)$ & -0.357 & $<0.00 I^{*}$ & 1.036 \\
CAT score & $-4.069(-7.602$ to -0.536$)$ & -0.306 & $0.025^{*}$ & 2.198 \\
Adjusted $R^{2}=0.445$ & & & & \\
\hline
\end{tabular}

Notes: *Statistical significance. Variables: age, FVC, FEV $, \mathrm{DL}_{\mathrm{co}}, \mathrm{RV} / \mathrm{TLC}, R_{20}, \mathrm{X}_{5}$, mMRC grade, SGRQ score, CAT score and CCQ score.

Abbreviations: $\beta$, regression coefficient; CAT, Chronic Obstructive Pulmonary Disease Assessment Test; CCQ, Clinical Chronic Obstructive Pulmonary Questionnaire; D6MWT, distance of 6-minute walk test; $\mathrm{DL}_{\mathrm{co}}$, diffusing capacity of the lungs for carbon monoxide; mMRC, modified Medical Research Council dyspnea scale; $R_{20}$, reactance at $20 \mathrm{~Hz}$; RV, residual volume; SGRQ, St George's Respiratory Questionnaire; TLC, total lung capacity; VIF, variance inflation factor; $\mathrm{X}_{5}$, reactance at $5 \mathrm{~Hz}$.

function and steps. These results support the relationship between lung function and steps.

In addition to the spirometry, other pulmonary function parameters presented varying degrees of relevance to the S6MWT. As can be seen from Table 3, $\mathrm{DL}_{\mathrm{CO}}$, an indicator of lung gas transfer that may be altered secondarily to impairment in small airways,$^{24}$ was positively correlated with S6MWT. It showed that COPD patients walk more steps with better diffusing capacity of the lungs. Prior studies revealed that an increase in RV is an early functional manifestation of COPD. ${ }^{25}$ Moreover, the study by Zeng et al suggested that RV was increased while TLC was only mildly elevated. ${ }^{26}$ However, in our study, neither RV nor TLC was significantly correlated with D6MWT or S6MWT. In contrast, the ratio of RV to TLC was significantly correlated with both D6MWT and S6MWT. The most possible explanation for the inconsistency is that the majority of the participants $(87.1 \%)$ in this study were in mild-moderate severity classification and the change in RV or TLC separately was too mild to be identified in the correlational analysis, while the ratio of them might be more sensitive in this population. Furthermore, from the results of S6MWT and IOS parameters, we can see that S6MWT was correlated with $\mathrm{X}_{5}$, but not $R_{20}$. It can be inferred that S6MWT is mainly influenced by peripheral airway function instead of proximal airway function, ${ }^{27,28}$ which is in accordance with the functional change of small airways in COPD. To summarize, S6MWT is correlated with various pulmonary function parameters measured by different methods and reflects the status of pulmonary function.

HRQoL questionnaires are commonly used in the quantity assessment of quality of life, therapeutic evaluation and have good consistency with COPD patients' exercise tolerance, with higher score or grade indicating worse functional status. ${ }^{29}$ The alternative questionnaires for assessment of COPD have respective advantages and disvantanges. ${ }^{30}$ The mMRC is a traditional and simple scale applied in the assessment of dyspnea. SGRQ is well validated and reliable. It is the most widely used HRQoL questionnaire in COPD, while it may be time-consuming to complete and is complex to score. CAT and CCQ are shorter and simpler questionnaires. CAT has been recommended to assess symptom burden and assist in guiding management by the GOLD guidements. ${ }^{11}$ CCQ is another valid questionnaire for COPD but was less well known than CAT, although it may have some advantages over CAT in some cases..$^{18}$ For this reason, we had adopted these four different questionnaires in this study. The results of the correlational analysis shown in Table 3 have demonstrated that both D6MWT and S6MWT were closely correlated with the score or grade of COPD questionnaires. COPD patients with respiratory symptoms in daily life also reflect in S6MWT.

Another point deserving consideration is that S6MWT, compared with D6MWT, may be more efficient in reflecting lung hyperinflation in patients with COPD. In this study, the results obtained from stepwise multiple linear regression analysis showed that mMRC grades, age and CAT score

Table 5 Stepwise multiple linear regression analysis for S6MWT

\begin{tabular}{l|l|l|l|l}
\hline Variable & Coefficient $(95 \% \mathbf{C I})$ & Standardized $\beta$ & $P$-value & VIF \\
\hline Intercept & $699.972(634.661-765.283)$ & & & \\
$\mathrm{DL}_{\text {co }}(\%$ predicted $)$ & $1.126(0.576-1.677)$ & 0.429 & $<0.001 *$ & 1.306 \\
CAT score & $-3.571(-5.812$ to -1.329$)$ & -0.334 & $0.002 *$ & 1.306 \\
Adjusted $R^{2}=0.417$ & & & \\
\hline
\end{tabular}

Notes: *Statistical significance; $\rho$ : correlation coefficient. Variables: IC, FVC, $\mathrm{FEV}_{1}, \mathrm{SPO}_{2}, \mathrm{DL}_{\mathrm{CO}}, \mathrm{RV} / \mathrm{TLC}, \mathrm{X}_{5}$, $\mathrm{mMRC}$ grade, SGRQ score, CAT score and CCQ score. Abbreviations: $\beta$, regression coefficient; CAT, Chronic Obstructive Pulmonary Disease Assessment Test; CCQ, Clinical Chronic Obstructive Pulmonary Questionnaire; $\mathrm{DL}_{\mathrm{co}}$, diffusing capacity of the lungs for carbon monoxide; IC, inspiratory capacity; mMRC, modified Medical Research Council dyspnea scale; RV, residual volume; S6MWT, steps of 6-minute walk test; SGRQ, St George's Respiratory Questionnaire; TLC, total lung capacity; VIF, variance inflation factor. 
were independent determinants of D6MWT. It was to some extent lined with the conclusion from study of Casanova et al, which had established that demographic characteristics such as age, height, weight and gender were the main determinants of D6MWT rather than pulmonary function parameters like $\mathrm{FEV}_{1} \cdot{ }^{31}$ This study by Casanova et al was performed in healthy subjects, while the findings still provide a reference for COPD. As demonstrated in our results, except for age, two indexes indicating quality of life, mMRC grade and CAT score, were remained in the final model for D6MWT. It may be that although the decline in pulmonary function is the underlying cause leading to exercise intolerance, respiratory symptoms, scores of HRQoLs questionnaires reflect the functional status more directly and relate much closer to D6MWT. Similar explanation may be applied in the results of S6MWT. In the final model for S6MWT, only CAT score and $\mathrm{DL}_{\mathrm{CO}}$ remained as the independent determinants. First, we can infer that among the various parameters, quality of life plays an important role in S6MWT. It further strengthens the link between quality of life and S6MWT. Second, as a result of small airway dysfunction, $\mathrm{DL}_{\mathrm{CO}}$ is altered in case of lung hyperinflation and emphysema. ${ }^{24,32}$ The results of multiple regression analysis had demonstrated that both D6MWT and S6MWT are primarily determined by HRQoL questionnaire score. However, S6MWT seems to be better in predicting lung hyperinflation in COPD when taking the results of correlational analysis and stepwise multiple regression analysis into comprehensive consideration, while further investigation and experimentation for it is recommended.

\section{Limitations}

There were some limitations in this study. First, as mentioned above, most of the participants in our study were within mild-moderate severity classification of COPD, therefore, the validity and efficiency of S6MWT should be checked in every grade of COPD. Second, since lung hyperinflation does not appear during the 6MWT in healthy subjects, the role of S6MWT in people without COPD is not clear. Additional work will be needed to clarify whether S6MWT is useful in identifying other pathophysiologic changes in the elderly.

\section{Conclusion}

Our study has shown that S6MWT correlates well with pulmonary function and quality of life in patients with COPD. S6MWT can be used as a novel index in the evaluation of functional status of COPD. More importantly, steps might give additional indication in addition to distance traveled about the risk of lung hyperinflation. Considerably more work will be needed to investigate the link between steps and hyperinflation in patients with COPD.

\section{Acknowledgments}

The authors would like to thank the participants, their families and the nurses of the Department of Respiratory Medicine, Zhujiang Hospital involved in this study. Jun-Kang Lan and Mei Zi are acknowledged for their assistance with data collection.

\section{Disclosure}

The author reports no conflicts of interest in this work.

\section{References}

1. Hartley RA, Barker BL, Newby C, et al. Relationship between lung function and quantitative computed tomographic parameters of airway remodeling, air trapping, and emphysema in patients with asthma and chronic obstructive pulmonary disease: a single-center study. J Allergy Clin Immunol. 2016;137(5):1413-1422.e12.

2. Habraken JM, van der Wal WM, Ter Riet G, Weersink EJ, Toben F, Bindels PJ. Health-related quality of life and functional status in end-stage COPD: a longitudinal study. Eur Respir J. 2011;37(2): 280-288.

3. Salzman SH. The 6-min walk test: clinical and research role, technique, coding, and reimbursement. Chest. 2009;135(5):1345-1352.

4. Waschki B, Kirsten AM, Holz O, et al. Disease progression and changes in physical activity in patients with chronic obstructive pulmonary disease. Am J Respir Crit Care Med. 2015;192(3):295-306.

5. Chandra D, Wise RA, Kulkarni HS, et al; NETT Research Group. Optimizing the 6-min walk test as a measure of exercise capacity in COPD. Chest. 2012;142(6):1545-1552.

6. Casanova C, Cote C, Marin JM, et al. Distance and oxygen desaturation during the 6-min walk test as predictors of long-term mortality in patients with COPD. Chest. 2008;134(4):746-752.

7. Pinto-Plata VM, Cote C, Cabral H, Taylor J, Celli BR. The 6-min walk distance: change over time and value as a predictor of survival in severe COPD. Eur Respir J. 2004;23(1):28-33.

8. Lahousse L, Verlinden VJ, van der Geest JN, et al. Gait patterns in COPD: the Rotterdam Study. Eur Respir J. 2015;46(1):88-95.

9. Mendoza L, Horta P, Espinoza J, et al. Pedometers to enhance physical activity in COPD: a randomised controlled trial. Eur Respir J. 2015; 45(2):347-354.

10. Guenette JA, Webb KA, O'Donnell DE. Does dynamic hyperinflation contribute to dyspnoea during exercise in patients with COPD? Eur Respir J. 2012;40(2):322-329.

11. Global Initiative for Chronic Obstructive Lung Disease. Global strategy for prevention, diagnosis and management of COPD (2018). Available from: https://goldcopd.org/gold-reports/. Accessed November 15, 2017.

12. Miller MR, Hankinson J, Brusasco V, et al. Standardisation of spirometry. Eur Respir J. 2005;26(2):319-338.

13. Guenette JA, Chin RC, Cory JM, Webb KA, O'Donnell DE. Inspiratory capacity during exercise: measurement, analysis, and interpretation. Pulm Med. 2013;2013:1-13.

14. MacIntyre N, Crapo RO, Viegi G, et al. Standardisation of the single-breath determination of carbon monoxide uptake in the lung. Eur Respir J. 2005;26(4):720-735.

15. Han MK, Muellerova H, Curran-Everett D, et al. GOLD 2011 disease severity classification in COPDGene: a prospective cohort study. Lancet Respir Med. 2013;1(1):43-50.

16. Weatherall M, Marsh S, Shirtcliffe P, Williams M, Travers J, Beasley R Quality of life measured by the St George's Respiratory Questionnaire and spirometry. Eur Respir J. 2009;33(5):1025-1030. 
17. Gupta N, Pinto LM, Morogan A, Bourbeau J. The COPD assessment test: a systematic review. Eur Respir J. 2014;44(4):873-884.

18. Kon SS, Dilaver D, Mittal M, et al. The Clinical COPD Questionnaire: response to pulmonary rehabilitation and minimal clinically important difference. Thorax. 2014;69(9):793-798.

19. ATS Committee on Proficiency Standards for Clinical Pulmonary Function Laboratories. ATS statement: guidelines for the six-minute walk test. Am J Respir Crit Care Med. 2002;166(1):111-117.

20. O'Donnell DE, Guenette JA, Maltais F, Webb KA. Decline of resting inspiratory capacity in COPD: the impact on breathing pattern, dyspnea, and ventilatory capacity during exercise. Chest. 2012;141(3):753-762.

21. O'Donnell DE, Casaburi R, Frith P, et al. Effects of combined tiotropium/olodaterol on inspiratory capacity and exercise endurance in COPD. Eur Respir J. 2017;49(4):1601348.

22. Marin JM, Carrizo SJ, Gascon M, Sanchez A, Gallego B, Celli BR. Inspiratory capacity, dynamic hyperinflation, breathlessness, and exercise performance during the 6-minute-walk test in chronic obstructive pulmonary disease. Am J Respir Crit Care Med. 2001;163(6): $1395-1399$.

23. Wibmer T, Rüdiger S, Kropf-Sanchen C, Stoiber KM, Rottbauer W, Schumann C. Relation of exercise capacity with lung volumes before and after 6-minute walk test in subjects with COPD. Respir Care. 2014; 59(11):1687-1695.

24. Harvey BG, Strulovici-Barel Y, Kaner RJ, et al. Risk of COPD with obstruction in active smokers with normal spirometry and reduced diffusion capacity. Eur Respir J. 2015;46(6):1589-1597.
25. Macklem PT. Therapeutic implications of the pathophysiology of COPD. Eur Respir J. 2010;35(3):676-680.

26. Zeng S, Tham A, Bos B, Jin J, Giang B, Arjomandi M. Lung volume indices predict morbidity in smokers with preserved spirometry. Thorax. Epub 2018 Jul 20.

27. Goldman MD, Saadeh C, Ross D. Clinical applications of forced oscillation to assess peripheral airway function. Respir Physiol Neurobiol. 2005;148(1-2):179-194.

28. Bickel S, Popler J, Lesnick B, Eid N. Impulse oscillometry: interpretation and practical applications. Chest. 2014;146(3):841-847.

29. Esteban C, Quintana JM, Aburto M, et al. Impact of changes in physical activity on health-related quality of life among patients with COPD. Eur Respir J. 2010;36(2):292-300.

30. Jones PW, Adamek L, Nadeau G, Banik N. Comparisons of health status scores with MRC grades in COPD: implications for the GOLD 2011 classification. Eur Respir J. 2013;42(3):647-654.

31. Casanova C, Celli BR, Barria P, et al; Six Minute Walk Distance Project (ALAT). The 6-min walk distance in healthy subjects: reference standards from seven countries. Eur Respir J. 2011;37(1):150-156.

32. Nambu A, Zach J, Schroeder J, et al. Relationships between diffusing capacity for carbon monoxide $\left(\mathrm{DL}_{\mathrm{CO}}\right)$, and quantitative computed tomography measurements and visual assessment for chronic obstructive pulmonary disease. Eur J Radiol. 2015;84(5):980-985.
International Journal of COPD

\section{Publish your work in this journal}

The International Journal of COPD is an international, peer-reviewed journal of therapeutics and pharmacology focusing on concise rapid reporting of clinical studies and reviews in COPD. Special focus is given to the pathophysiological processes underlying the disease, intervention programs, patient focused education, and self management protocols.

\section{Dovepress}

This journal is indexed on PubMed Central, MedLine and CAS. The manuscript management system is completely online and includes a very quick and fair peer-review system, which is all easy to use. Visit $\mathrm{http}: / /$ www.dovepress.com/testimonials.php to read real quotes from published authors. 